\title{
Night Blindness
}

National Cancer Institute

\section{Source}

National Cancer Institute. Night Blindness. NCI Thesaurus. Code C34850.

Inability to see clearly in dim light. 